\title{
Insights into the yellowing of drying oils using fluorescence spectroscopy
}

\author{
L. K. Cairns and P. B. C. Forbes ${ }^{*}$ (1)
}

\begin{abstract}
Drying oils are commonly used in paintings and are prone to yellow discolouration from aging. Although there are numerous studies aimed at understanding their chemical composition, the yellowing process and its effects on the underlying fluorescence emission of the oils are not fully understood. In this study, four different commercially available oils, namely linseed, water-miscible linseed-, stand- and poppy seed oil, were analysed using UV-visible absorption and fluorescence spectroscopy. Both liquid and cured, solid film oils were analysed. Liquid oils show a structured absorption pattern, of which only two weakly absorbing peaks ( $\lambda_{\mathrm{ex}} 300$ and $315 \mathrm{~nm}$ ) result in fluorescence emission $\left(\lambda_{\mathrm{em}} 330\right.$ and $\left.410 \mathrm{~nm}\right)$. The solid film lacks the structured pattern seen in the liquid oil's absorption spectrum, showing instead a broad absorption peak. At an excitation wavelength $\left(\lambda_{\mathrm{ex}}\right)$ of $365 \mathrm{~nm}$ the cured film normally fluoresces at $\lambda_{\mathrm{em}} 440 \mathrm{~nm}$ but is seen to shift to $\lambda_{\mathrm{em}} 550 \mathrm{~nm}$ as a result of yellowing. Artificial aging techniques, applied to the oils in order to cause a large degree of yellow discolouration, were seen to induce a large bathochromic shift in their fluorescence spectra. A correlation between the degree of discolouration and the shift in fluorescence is demonstrated, giving rise to a quantitative method that can be used to monitor the yellow discolouration. By non-destructively quantifying the degree of discolouration, colour-reconstruction of paintings could be performed to identify what the paintings looked like before degradation.
\end{abstract}

Keywords: Drying oils, Yellowing, Fluorescence spectroscopy, Absorption spectroscopy, Artificial aging, Painting

\section{Introduction}

Drying oils have been used in paintings as a binding medium since the 15 th century [1-3]. Their ability to dry and form a solid film under ambient conditions [4] can be attributed to the unsaturation in oils, which facilitates polymerization [2]. The properties of each oil are largely dependent on the type of fatty acids present; for example, oils with a high unsaturated fatty acid content dry faster but have a greater tendency to yellow with age $[3,5-8]$. Linseed-, poppy seed-, as well as walnut- or safflower-oil, which are commonly used in paintings, contain high concentrations of the unsaturated fatty acids, linolenic and linoleic acid (Table 1) [9]. Linseed oil is extracted from the flax plant (Linum usitatissimum), which contains

*Correspondence: patricia.forbes@up.ac.za

Department of Chemistry, Faculty of Natural and Agricultural Sciences,

University of Pretoria, Pretoria 0002, South Africa large amounts of unsaturated fatty acids [3]. Water-miscible linseed oil contains the same oil content as linseed oil with an added emulsifier that increases hydrophilicity and thereby allows for water to rinse paint brushes, instead of toxic organic solvents [2]. Water-miscible oil paints were introduced to the artist's palette around the 1990s [10] although oil and water paint mixtures had previously been used for centuries [11]. Stand oil is a form of linseed oil that has been pre-polymerised through heat treatment. Poppy seed oil is extracted from Papaver somniferum seeds and has a lower degree of unsaturation [1]. There are numerous manufacturing processes, and thus numerous historical formulations, for the production of drying oils $[12,13]$, however, the oils used for this study are representative of the 21st century artist's palette, and therefore commercially available drying oils used were tested.
Springer Open

(C) The Author(s) 2020. This article is licensed under a Creative Commons Attribution 4.0 International License, which permits use, sharing, adaptation, distribution and reproduction in any medium or format, as long as you give appropriate credit to the original author(s) and the source, provide a link to the Creative Commons licence, and indicate if changes were made. The images or other third party material in this article are included in the article's Creative Commons licence, unless indicated otherwise in a credit line to the material. If material is not included in the article's Creative Commons licence and your intended use is not permitted by statutory regulation or exceeds the permitted use, you will need to obtain permission directly from the copyright holder. To view a copy of this licence, visit http://creativeco mmons.org/licenses/by/4.0/. The Creative Commons Public Domain Dedication waiver (http://creativecommons.org/publicdomain/ zero/1.0/) applies to the data made available in this article, unless otherwise stated in a credit line to the data. 
Table 1 The fatty acid content of drying oils typically present in paintings $[6,14,15]$

\begin{tabular}{llllll}
\hline Fatty acids & & Linseed oil (\%) & Poppy seed oil (\%) & Walnut oil (\%) & Safflower oil (\%) \\
\hline Palmitic acid & $($ C16:0*) & $6-8$ & $8-12$ & $3-7$ & $5.5-7$ \\
Stearic acid & $($ C18:0) & $3-6$ & $2-3$ & $0.5-3$ & $2-3$ \\
Oleic acid & $($ C18:1) & $14-24$ & $12-17$ & $9-30$ & $10-35$ \\
Linoleic acids & $($ C18:2) & $14-19$ & $55-65$ & $57-76$ & $55-81$ \\
Linolenic acid & $($ C18:3) & $48-60$ & $3-8$ & $2-16$ & $0-1$ \\
\hline
\end{tabular}

* C16 indicates the number of carbons in the chain (in this case, 16 carbons), while the second number indicates the degree of unsaturation (number of double bonds)

The discolouration of paintings is of concern, as we can no longer appreciate a painting for its original colours. There are several factors that lead to discolouration such as the degradation of pigments [16-19], formation of lead soaps [20-23], the yellowing of varnish [24, 25] and oil binding media [26-28]. This study focuses on the discolouration of drying oils. Drying oils are clear or faintly coloured when applied to paintings, but gradually develop a strong yellow discoloration over time. This discoloration is an unavoidable property of oil paint [2]. The basis of the yellow colour remains poorly understood, despite the wide range of reported chemical compounds that are suspected to play a role in this regard [2, 4, 26-32]. The currently accepted view is that the yellowing is a result of two compounds, one which reflects yellow light, and another which fluoresces yellow, however, their identities are still unknown. Together these two compounds are thought to make up the characteristic intense yellow colour [29].

Although little is known of the yellowing of oils, there are a few trends associated with the yellowing process. Firstly, a high degree of unsaturation in the oil leads to significant discolouration. Therefore, linseed oil will yellow to a greater extent than poppy seed oil, which is less unsaturated [2,33]. Secondly, the yellowing has been found to be accelerated by certain metal-containing pigments, such as lead white, copper carbonate and various cobalt pigments [26, 33-36]. Thirdly, the discolouration can be bleached and can go through cycles of yellowing and bleaching, depending on the storage conditions; light exposure reverses the yellowing, while dark conditions promote yellowing, which suggests that photodegradation reactions reverse the discolouration $[26,36]$. Lastly, there is a correlation between the fluorescence of drying oils and the degree of yellowing: fluorescence emission shifts from blue to green as the samples yellow [26, 29, 37].

In contrast to yellowing, the fluorescent property of oils is beneficial as it can aid in diagnostic tests that identify areas of previous restoration [38-40].
Fluorescence imaging of a painting is obtained by illuminating its surface with ultra-violet (UV) light and observing the visible-light fluorescence. The development of the LED micro-spectrofluorometer (LED $\mu \mathrm{SF}$ ) and other handheld spectrofluorometers has allowed for more sophisticated spectroscopic analysis [41-45]. The fluorescence of a painting can now be measured as a spectrum, with exact emission wavelengths, instead of a single colour determined subjectively by the eye.

This study aimed to identify changes in yellowing, during the curing of drying oils, through the use of highsensitivity fluorescence spectroscopy. Four commercially available drying oils were exposed to different light conditions (sun light, UV-light and dark) during the curing process, and afterwards were exposed to artificial aging mechanisms (UV-light and an ammonia vapour chamber respectively) to accelerate yellowing. By correlating the fluorescence changes with the degree of yellowing, digital colour corrections can be performed on paintings without the need to sample. This allows a viewer to see what the colours would likely have looked like before agerelated degradation occurred.

\section{Experimental methods \\ Sample preparation}

Solid film samples were prepared by painting the drying oils onto glass microscope slides which were allowed to dry in order for film formation to occur. Four drying oils were tested: linseed oil (LO), water-miscible linseed oil (WLO), stand oil (SO) and poppy seed oil (PO), all from Winsor \& Newton, UK. The solid samples were naturally aged for 2 years under ambient temperature $\left(20 \pm 5{ }^{\circ} \mathrm{C}\right)$ and humidity $(50 \pm 20 \% \mathrm{RH})$ in a drawer. Fluctuations in temperature and humidity are resultant of seasonal changes. Liquid samples were prepared by dissolving $10 \mu \mathrm{L}$ of the liquid oil in $1000 \mu \mathrm{L}$ of ethanol $(99.9 \%$ from ACE, South Africa) or other solvent. The solvents used were diethyl ether (90\% SAR Chem, South Africa), ethanol, dichloromethane and toluene at $95 \%$ purity (ACE, South Africa). Acetonitrile, hexane and toluene were HPLC grade (Sigma Aldrich, South Africa), while 
cyclohexane and chloroform were $99.5 \%$ purity (RadChem, South Africa).

Solid-liquid extraction was performed on the dried oils by placing $0.500 \mathrm{mg}$ cured oil into a vial with 1000 $\mu \mathrm{L}$ ethanol. The vial was then ultrasonicated at a high frequency $(50 \mathrm{~Hz})$ for $30 \mathrm{~min}$ using a Scientech ultrasonic cleaner (Labtec, South Africa). Additionally, the solid film on a microscope slide was extracted through Soxhlet extraction, using $100 \mathrm{~mL}$ ethanol for $24 \mathrm{~h}$. The liquid extract was yellow coloured, while the remaining solid film was severely cracked.

Samples for artificial aging were prepared by painting the drying oils onto microscope slides covered with pieces of painter's canvas to facilitate the accelerated aging with minimal alligator-skin formation (wrinkling of paint caused by rapid drying of the upper layers of the oil). These samples were solely used for fluorescence studies and to monitor the changes in fluorescence during artificial aging.

\section{Artificial ageing and curing}

Artificial ageing was achieved by exposing the samples to elevated temperatures, artificial light and a saturated chamber of ammonia. An ammonia chamber was prepared to induce yellowing. The wooden chamber $(25 \times 25 \times 60 \mathrm{~cm})$ was sealed with rubber and had a glass window through which the colour change could be monitored. A beaker containing $40 \mathrm{~mL}$ of $25 \%$ ammonia solution was placed in the chamber to saturate the air with ammonia vapour. Two main sets of samples were treated, the first set of samples was exposed to various ageing environments while wet, and the samples were cured under the ageing conditions. The second set was first cured in a dark chamber for 3 months after which the samples were exposed to the artificial ageing conditions.

Within each set, there were various times for which a sample was exposed to the ageing conditions. The first set of 44 samples were cured under the ageing conditions: two samples for each oil (a total of 8 samples) were cured exposed to an elevated temperature $\left(80^{\circ} \mathrm{C}\right)$, with one sample exposed for $3 \mathrm{~h}$ and the other for $6 \mathrm{~h}$. Another set of 5 samples per oil were cured for either 24 h, 1 week, 2 weeks, 3 weeks or 1 month under a UVC lamp $(254 \mathrm{~nm})$, thereafter they were placed in a dark drawer. Another set of 4 samples for each oil were cured in the ammonia chamber under ambient indoor light conditions, for either $24 \mathrm{~h}, 1$ week, 2 weeks or 1 month, and thereafter placed in a dark drawer.

The second set of 24 samples was cured in a dark drawer for 3 months before exposing the samples to the different ageing conditions. Two samples for each oil were placed under the UV lamp for either $24 \mathrm{~h}$ or 1 week, and thereafter were placed in a dark drawer. Another set of 4 samples for each oil were placed in the ammonia chamber for either $24 \mathrm{~h}, 1$ week, 2 weeks or 1 month and thereafter were stored in a dark drawer prior to analysis.

\section{Absorption and fluorescence spectroscopy}

During both absorption and fluorescence studies, liquid samples were held in $10 \mathrm{~mm}$ quartz cuvettes, which were washed between each experiment using 10\% Piranha solution. For fluorescence studies, painted samples on microscope slides were analysed in a slide-holder at a $30^{\circ}$ angle to the incident light, to prevent reflected incident light from entering the detector.

\section{UV-visible light absorption spectrophotometer}

UV-visible absorption was done using a Cary 100-Bio UV-visible spectrophotometer (Varian, USA) equipped with a visible light source lamp and a deuterium lamp, which switched at $350 \mathrm{~nm}$. Scans were measured from 800-200 nm with a Czerny-Turner $0.28 \mathrm{~m}$ monochromator equipped with a R928 photomultiplier tube (PMT) detector.

\section{Fluorescence spectrophotometer}

Fluorescence measurements were made on a Fluoromax-4 Spectrofluorometer (Horiba, Japan) with a continuous $150 \mathrm{~W}$ xenon lamp and photodiode detector. A single monochromator was used for excitation and emission wavelengths, with a slit width of $5 \mathrm{~nm}$ for both the light source and the detector.

\section{Chromatographic fractionation}

Samples were analysed using a 1260 Infinity binary highperformance liquid chromatography (HPLC) system (Agilent, USA), equipped with an Agilent 1260 auto sampler and a XSelect ${ }^{\circledR}$ HSS T3 $5 \mu \mathrm{m}(4.6 \mathrm{~mm} \times 150 \mathrm{~mm})$ reverse phase column (Waters, South Africa). The 1260 Infinity Photodiode-Array Detector (PDA) (Agilent, USA) used a deuterium lamp (wavelength range 190 to $640 \mathrm{~nm}$ ), with optical slit of $4 \mathrm{~nm}$. Chromatograms were monitored using select wavelengths: 240, 260, 280, 300, 315 and $400 \mathrm{~nm}$.

Separation was achieved by means of a reverse phase step gradient using acetonitrile (A) and water (B) both with $0.1 \%$ formic acid, at a $4.00 \mu \mathrm{L} / \mathrm{min}$ flow rate. Initial conditions were: $20 \%$ A: $80 \%$ B, ramped up to $100 \%$ A within $12 \mathrm{~min}$, held constant for $14 \mathrm{~min}$. Thereafter, the gradient was rapidly changed back to the initial conditions of $20 \% \mathrm{~A}$ in $2 \mathrm{~min}$ and then held constant for $2 \mathrm{~min}$. The HPLC system was fitted with a re-usable online micro solid phase extraction ( $\mu \mathrm{SPE})$ collection unit. Two fractions were manually collected onto the HysphereGP $20 \times 2 \mathrm{~mm} \mu \mathrm{SPE}$ cartridges (Spark Holland, The 
Netherlands) and were then eluted using HPLC grade methanol (Sigma Aldrich, South Africa).

\section{Results and discussion}

UV-absorption and fluorescence spectroscopic changes due to curing

The absorption spectra of drying oils were recorded for the liquid oils (dissolved in ethanol) and the solid films. There were few differences in the absorption between the four different drying oils in solution (Fig. 1). All oils have two strongly UV-absorbing peaks, one between 200 and $215 \mathrm{~nm}$ while the second, less intense peak occurred at $230 \mathrm{~nm}$. The absorption at $200-215 \mathrm{~nm}$ indicates the presence of polyenes and unsaturated $\alpha, \beta$-ketones $[46$, 47]. Between 250 and $350 \mathrm{~nm}$, the oils show weak absorptive peaks indicating the presence of an unconjugated chromophore containing a heteroatom [46].

The spectra of linseed oil (LO) and water-miscible linseed oil (WLO) are the most similar: both show peaks at about 280, 300 and $315 \mathrm{~nm}$, while the peak at $269 \mathrm{~nm}$ (in the presence of another peak at $315 \mathrm{~nm}$ ) is characteristic of WLO. The spectral similarity between LO and WLO are not surprising, considering the fact that WLO is LO with an emulsifier that increases its solubility in water, but other than that, the two are chemically identical [48]. Poppy seed oil (PO) has three additional moderately absorbing peaks, at 258, 269 and $279 \mathrm{~nm}$, of which the first peak is characteristic of $\mathrm{PO}$ and does not appear in either LO or WLO. Stand oil (SO) does not have any peaks in the range between 250 and $350 \mathrm{~nm}$, suggesting that the compounds that would normally give rise to peaks in this area may have reacted during the prepolymerization process [3]. In contrast to the absorption

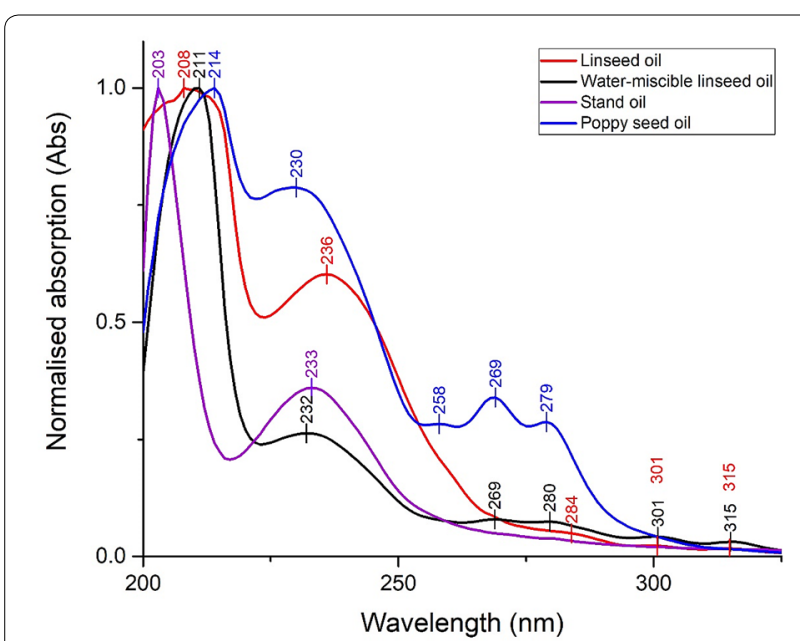

Fig. 1 Absorption spectra of the liquid drying oils dissolved in ethanol. All spectra were blank corrected and normalised to 1 spectra of samples in solution, the cured films show only a broad peaked absorption at $300 \mathrm{~nm}$ (Fig. 2).

The absorption patterns give an indication of the excitation wavelength which will result in optimal fluorescence. Of the 5 distinct absorption peaks in liquid linseed oil, only two resulted in fluorescence. Excitation $\left(\lambda_{\text {ex }}\right)$ at $300 \mathrm{~nm}$ gives a two-peaked fluorescence signal (Fig. 3) while excitation at $315 \mathrm{~nm}$ gives only one. The emission at $330 \mathrm{~nm}$ only appears upon excitation at $300 \mathrm{~nm}$, while the peak at $410 \mathrm{~nm}$ appears when both excitation wavelengths $\left(\lambda_{\mathrm{ex}} 300\right.$ and $\left.315 \mathrm{~nm}\right)$ are used. Although $\mathrm{PO}$ and SO show no absorption at 300 and $315 \mathrm{~nm}$, both

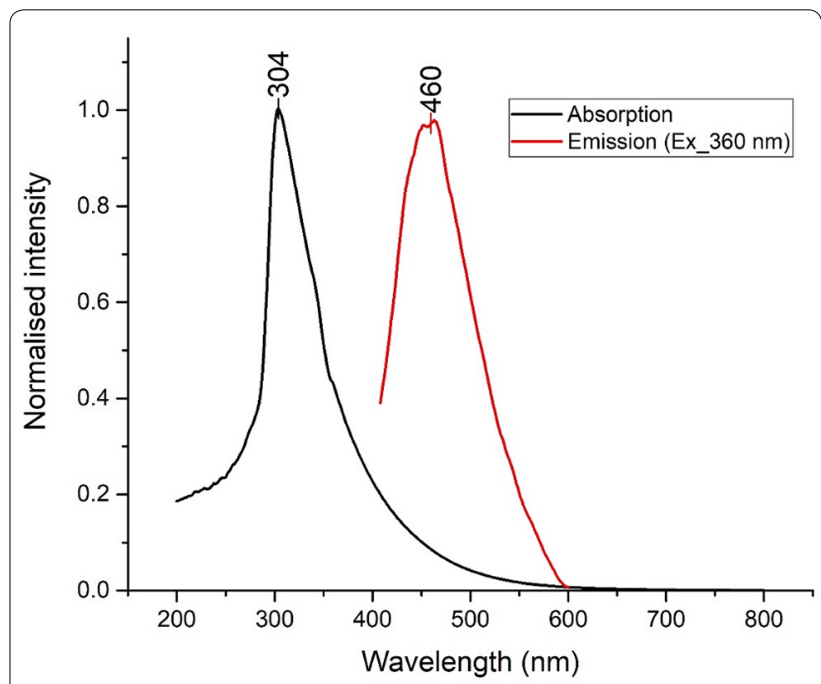

Fig. 2 Fluorescence and absorption spectra of cured drying oils (once they formed a solid film). All oils showed the same peaks

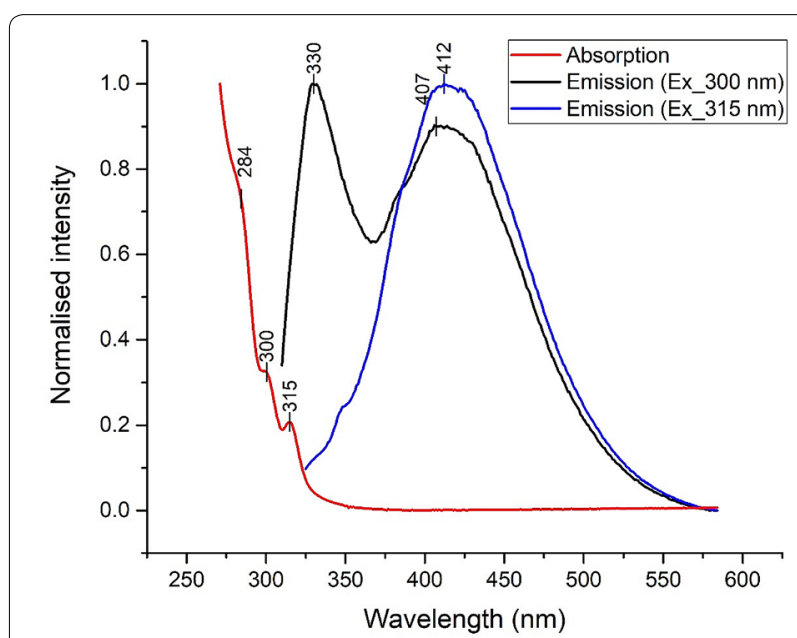

Fig. 3 Absorption spectra of liquid linseed oil dissolved in ethanol (red) and corresponding fluorescence spectra, at an excitation wavelength $\left(\lambda_{\mathrm{ex}}\right) 300 \mathrm{~nm}$ (black) and $\lambda_{\mathrm{ex}} 315 \mathrm{~nm}$ (blue), respectively 
excitation wavelengths give the same fluorescence spectra as LO, and no additional fluorescence is observed.

Curing of the liquid-phase samples shifts the fluorescence from a weak ultraviolet emission (330 and $\sim 410 \mathrm{~nm}$ ) to a strong blue-green fluorescence in the solid film (Fig. 2). Bathochromic shifts (shifts to longer wavelengths) of such magnitude (50 to $100 \mathrm{~nm}$ ) are not uncommon and are often induced through changes in solvent polarity or hydrogen bonding [49]. Drying oils are cured through an auto-oxidation process which results in a change from a non-polar liquid oil to a polar polymer network [47]. To determine whether the bathochromic shift is a result of a polarity change, the Lippert equation can be used [49]. The equation assumes that no external factors interact with the fluorophore; additional interactions, such as hydrogen bonding or the formation of charge-transfer states, can be detected as deviations from this generalised state. In this case, applying the Lippert equation reveals no trend, indicating that the bathochromic shift is not a result of polarity changes (Additional file 1: Figure S1). Interestingly, the intensity of the two fluorescent peaks from the liquid samples is found to be solvent-dependent. Polar solvents result in both peaks having similar fluorescence intensities, whereas non-polar solvents halve the intensity of the first peak $(330 \mathrm{~nm})$ while the second peak $(410 \mathrm{~nm})$ remains at a reasonably constant intensity (Additional file 1: Figure S2).

The solvent-extractable components of the cured oil were analysed. The cured linseed oil film does not fully dissolve, regardless of the polarity of solvent used. Ethanol proves to yield the largest fluorescent intensity, confirming literature reports of ethanol being the most efficient solvent for extracting compounds from LO [50].
The extracts (Soxhlet- and ultrasonic-extractions) show a single absorption peak (315 $\mathrm{nm}$ ) which corresponds to that of the liquid oil, with the absence of the absorption peak at $300 \mathrm{~nm}$. The yellow extract was found to be stable with no noticeable colour changes after a period of a month. The fluorescence of the extract was tested directly after extraction and a month later to determine whether any degradation or instability had occurred, and the fluorescence was found to remain stable at $418 \mathrm{~nm}$.

The fluorescence peak of the extract at $418 \mathrm{~nm}$ corresponds to liquid LO and not its solid phase (Table 2). The remaining solid film remains strongly fluorescent under UV light, and shows no change in fluorescence emission. However, the film was severely cracked and could not be used for further absorption studies.

The ethanol extract of the cured oil was fractionated using high performance liquid chromatography (HPLC) coupled to a photodiode array detector (PDA) to identify the most optically active fractions. The chromatograms were monitored using a range of absorption wavelengths, as no peaks were seen using $315 \mathrm{~nm}$. Interestingly, only $280 \mathrm{~nm}$ show peaks on the chromatogram. Two fractions were collected corresponding to the detection of two large absorption peaks, at $12.8 \mathrm{~min}$ (fraction 1) and 13.2 min (fraction 2; Additional file 1: Figure S3) respectively. These two peaks were found upon analysis of both the liquid oil and cured oil extracts.

Both fraction 1 and 2 have the same strong absorption peak at $275 \mathrm{~nm}$, while fraction 1 has an additional peak at $232 \mathrm{~nm}$ (Fig. 4 and Table 2). The fluorescence emission of the two fractions are both at $315 \mathrm{~nm}\left(\lambda_{\text {ex }} 275 \mathrm{~nm} ; \Delta\right.$ $40 \mathrm{~nm}$ ), which does not correspond to that of the liquid or cured oil (Table 2). This suggests that the absorption at $315 \mathrm{~nm}$ is highly dependent on the oil matrix, and that

Table 2 Shifts in absorption and emission bands of the liquid sample, cured film, ethanol extract and the oil film after ethanol extraction, as well as the two purified HPLC fractions in methanol

\begin{tabular}{|c|c|c|c|}
\hline & $\begin{array}{l}\text { Absorption band maximum; } \lambda_{\mathrm{ex}} \\
(\mathrm{nm})\end{array}$ & $\begin{array}{l}\text { Emission band maximum; } \lambda_{\mathrm{em}} \\
(\mathrm{nm})\end{array}$ & Stokes shift; $\Delta(\mathrm{nm})$ \\
\hline \multirow[t]{5}{*}{ Liquid } & 207 & - & - \\
\hline & 236 & - & - \\
\hline & 283 & - & - \\
\hline & 300 & 329,412 & 29,112 \\
\hline & 315 & 412 & 97 \\
\hline Cured oil film & 300 & $445 \rightarrow 550^{*}$ & $145 \rightarrow 250^{*}$ \\
\hline Ethanol extract from cured oil & 318 & 418 & 100 \\
\hline Cured oil film after ethanol extraction & - & 445 & - \\
\hline \multirow[t]{2}{*}{ Fraction 1} & 232 & - & - \\
\hline & 275 & 315 & 40 \\
\hline Fraction 2 & 275 & 315 & 40 \\
\hline
\end{tabular}

* Fluorescence of cured oil changes upon ageing; discussed in "Artificial ageing" section 


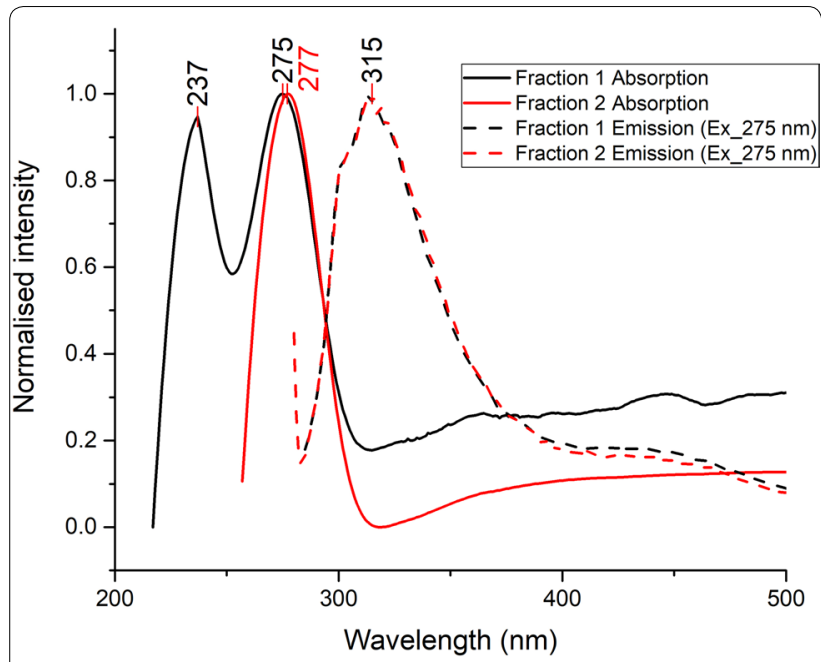

Fig. 4 Absorption and fluorescence spectra of the most optically active fractions in linseed oil collected by HPLC-PDA-SPE

once the compounds are isolated, they show different excitation spectra. The fractions have a smaller Stokes shift than the liquid oil and the extract of cured oil, suggesting that the isolated compound is not the same as the compound that results in the fluorescence in the cured oil.

The preceding findings indicate that the compound giving rise to the first peak in liquid oil, at $\lambda_{\mathrm{ex}} 300 \mathrm{~nm} ; \lambda_{\mathrm{em}}$ $330 \mathrm{~nm}$, reacts as part of a polymer structure, and cannot be extracted. While the second compound, which gives rise to the second peak at $\lambda_{\mathrm{ex}} 315 \mathrm{~nm} ; \lambda_{\mathrm{em}} 410 \mathrm{~nm}$, remains unreacted in the cured oil, and emits only in solution. This could be interpreted to mean that the first peak contributes significantly to the strong blue fluorescence in the solid phase, which is stabilised during the polymerization process. This is commonly seen in nonconjugated polymer dots (NCPDs) where poorly fluorescent subfluorophores (heteroatom-containing double bonds; $\mathrm{C}=\mathrm{O}, \mathrm{C}=\mathrm{N}, \mathrm{N}=\mathrm{O}$ ) are enhanced through chemical linking or physical immobilisation [51]. Subfluorophores have intrinsically weak fluorescence, although it increases drastically with vibrational and rotational restriction. NCPDs have characteristic blue fluorescence and can develop a bathochromic shift when the electron density over the subfluorophore increases [52]. This bathochromic shift in drying is seen as the oil ages (as is further explained in "Artificial ageing" section).

\section{Artificial ageing}

Oils that were cured under UVC light $(254 \mathrm{~nm})$ showed immediate alligator skin formation and bleaching. The accelerated curing of oils exposed to UV light is due to the radical mechanism of curing which is initiated through light [52-54]. After exposure to UV light, the cured samples were placed in dark drawers, which visibly accelerated the yellowing of the oils (Fig. 5). As the samples aged, the fluorescence was measured in weekly intervals, for 2 months, and then monthly, for another 6 months. It is important to note that the ageing methods employed in this study were extreme exposure to light and dark, which does not represent the typical light conditions in museums. However, the dark conditions could represent the storage areas of museum where paintings will reside for months in the dark. The extreme light conditions accelerated all ageing reactions, which allowed for a short-term monitoring of fluorescence changes $[55,56]$.

With aging, a bathochromic shift is observed, which can be related to the yellowing effect (Fig. 6) [29, 39, 57-59]. The fluorescence emission maximum is initially at $460 \mathrm{~nm}$ but increases exponentially to $500-510 \mathrm{~nm}$ (a green colour), where it reaches a plateau (Fig. 7). An additional few months in the dark drawer increases the emission further to $550 \mathrm{~nm}$, imparting a yellow-green fluorescent colour (Additional file 1: Figure S4) to the sample. This is the general trend for samples cured under ultra-violet light. The formation of a plateau of constant emission indicates that there are two processes that cause the bathochromic shift. This confirms that oil paintings are not only continuously changing, but that there are multiple steps in the process that can occur over a period of months.

Drying oils that have been cured under ambient conditions (dark drawer at $20 \pm 5{ }^{\circ} \mathrm{C}$ and $50 \pm 20 \% \mathrm{RH}$ ) and then exposed to UV light after 3 months, show a different trend. Instead of reaching a plateau, a steadily increasing emission wavelength is observed, that passes from blue $(450-490 \mathrm{~nm})$, to green $(495-570 \mathrm{~nm})$ and to yellow (570-590 nm) fluorescence (Fig. 7). Samples that were cured in sunlight did not show any yellowing, and thus no changes in fluorescence. Aging under dark conditions causes discolouration, however, without sunlight or UVlight exposure, the discolouration takes much longer to develop. Natural aging can take up to 2 years to develop the same kind of discolouration that UV-light causes within 2 weeks.

The curing of drying oils in an ammonia chamber appears to inhibit the drying process, as the oils appeared to be as viscous as when they were initially applied. Drying oils that were cured in the dark showed some degree of drying within the same time frame as those kept in the ammonia chamber. Interestingly, uncured samples that were in the ammonia chamber for a longer period (1 month) showed accelerated yellowing once cured (after 4 months), while a short exposure time to ammonia does not affect the yellowing rate (Fig. 8). This could be because the ammonia is absorbed into the liquid, and 


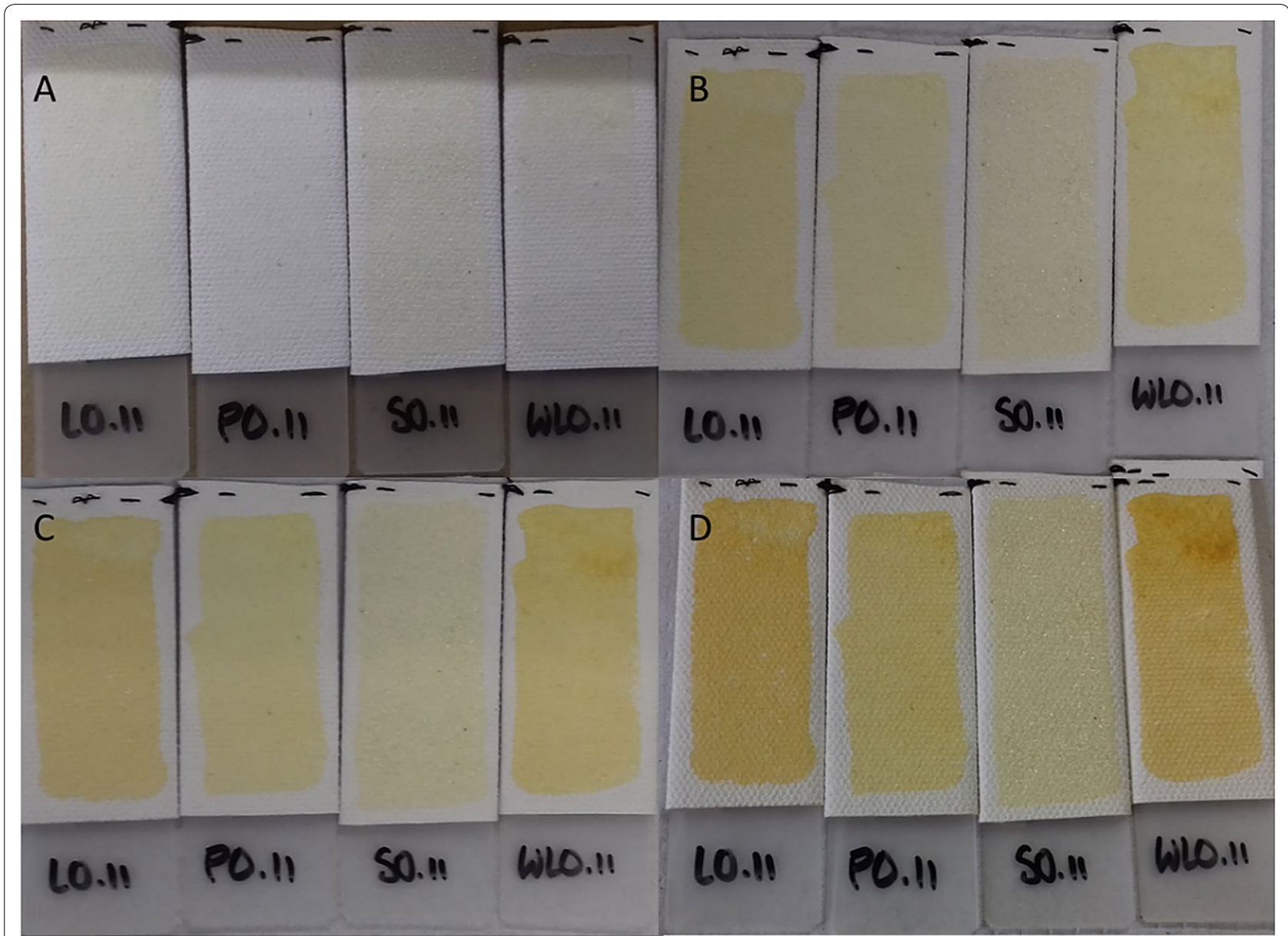

Fig. 5 Yellowing of oils after $24 \mathrm{~h}$ of UV-light exposure, and subsequent storage in a dark drawer. The colour changes that occur over time are evident, from freshly painted oil (a), to 7 days (b), 14 days (c) and 4 months (d) of storage in the dark drawer

when the oils are cured, the ammonia becomes more readily available, which accelerates the rate of yellowing.

When the ambiently cured oils were placed (after 3 months of curing) in the ammonia chamber, the colour of the oil changed rapidly. Within $24 \mathrm{~h}$, the saturated ammonia chamber changed all four oils to an orangebrown colour. The ammonia chamber caused the same degree of yellowing as UV-light treatment did, but within a 24-h period, rather than a month (Fig. 8). This supports the hypothesis that the presence of ammonia is a main cause for the acceleration of the yellowing found in oil paintings [29], and thus stresses the need to use ammonia-free cleaning products in museum environments. Previous studies have suggested the formation of a fluorescent aminoenamine in oils, which is responsible for bathochromic shifts [29, 30]. However, recent studies have shown that these isolated adducts may in fact not be fluorescent [60]. It is, however, still possible that the nitrogen-containing vapours are responsible for the colour changes through the formation of a fluorescent compound, as there have been studies that demonstrate through infrared spectroscopy that the nitrogen content in drying oils increases as the oils age [32].

All stages of yellowing (identified by green and yellow fluorescence) were reversible in sunlight within $8 \mathrm{~h}$ of exposure, as is evident from the hypsochromic shift in fluorescence (Fig. 9). The yellowing is reversed in neither 254 nor $365 \mathrm{~nm}$ UV light (in a 24-h period), indicating that a broad light spectrum is needed to reverse the yellowing effects. Artificial aging under UV light accelerates the rate of yellowing but yellowing cannot be used as a measure of age as the painting can be bleached. A bleached painting will thereby appear younger while a freshly painted portrait might look old if stored in the dark. The yellowing cycle can be repeated numerous times and still undergo the same degree of yellowing and bleaching [8], the bleaching process is faster than the yellowing. This suggests a hysteresis curve, in which the extent of yellowing is related to the light exposure history of the painting. Previous studies that have monitored the 


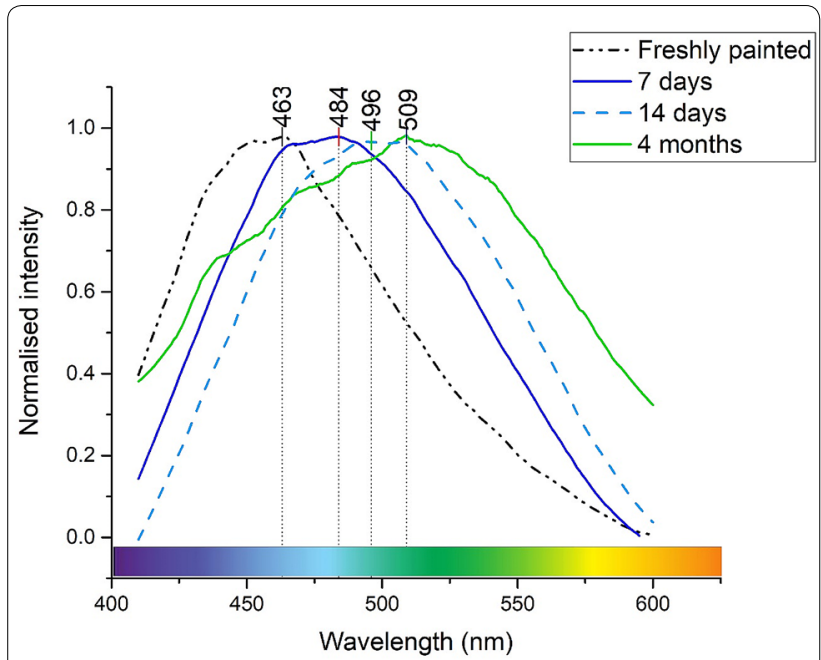

Fig. 6 Changes in the fluorescence spectrum of aged linseed, exposed for $24 \mathrm{~h}$ to $\mathrm{UV}$ light and subsequently stored in a dark drawer. The excitation wavelength for all measurements was $360 \mathrm{~nm}$. The bathochromic shift (to a longer emission wavelength) is a result of yellowing as the sample ages

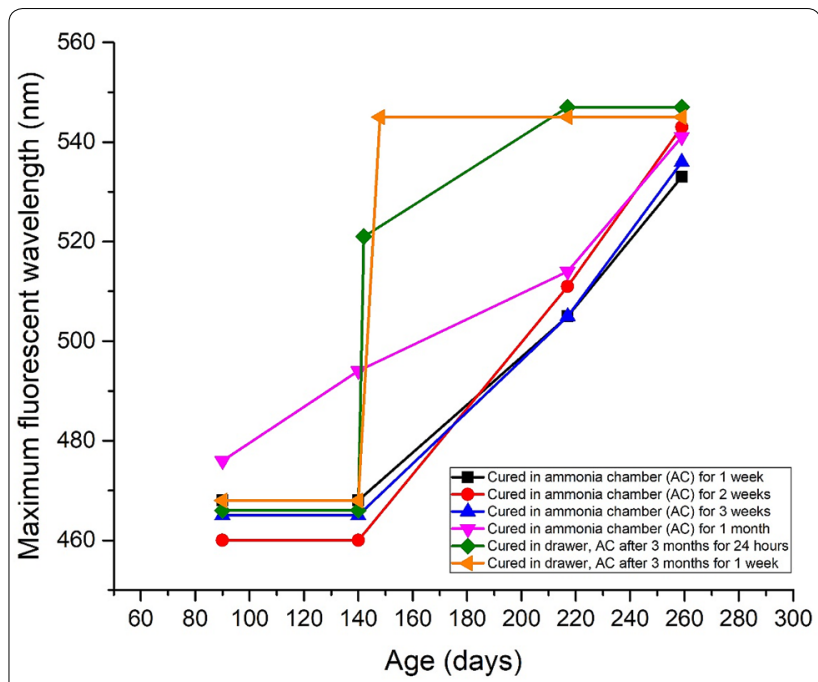

Fig. 8 Rate of fluorescent changes of linseed oil after exposure to ammonia vapor, and subsequent storage in a dark drawer. The excitation wavelength was $360 \mathrm{~nm}$ for all measurements

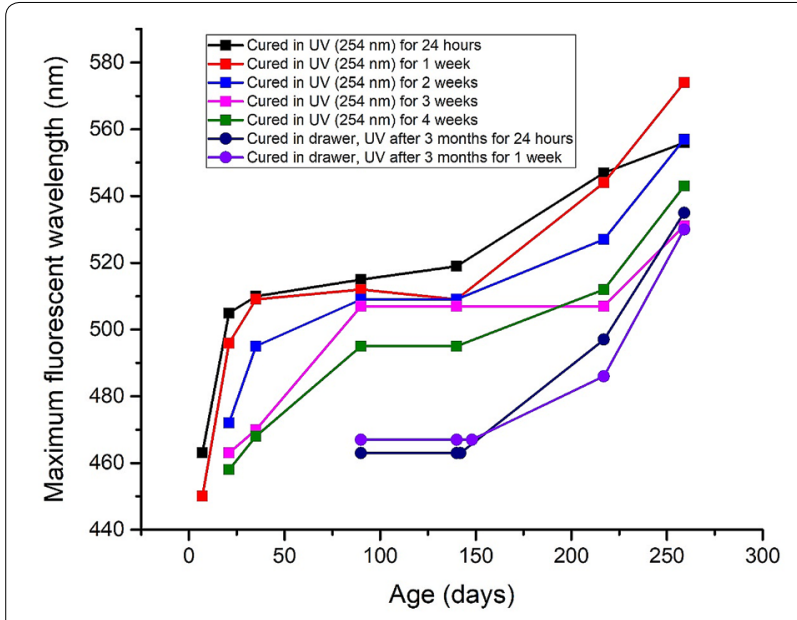

Fig. 7 Rate of the fluorescence emission maximum changes of linseed oil with various exposure times to UV light ( $254 \mathrm{~nm}$ ) and then subsequent storage in dark drawers. The excitation wavelength was $360 \mathrm{~nm}$ for all measurements

discolouration over several years have found a repetitive nature of the yellowing and bleaching of oils $[33,61]$. Even though the bleaching is cyclic, it is important to stress that it is a photodegradation reaction and therefore affects the integrity of the paint and should be avoided.

Although all drying oils showed a similar trend in their rate of yellowing, they did not all yellow to the same extent. Linseed oil (both LO and WLO) shows the most intense colour changes, while poppy seed oil yellows less,

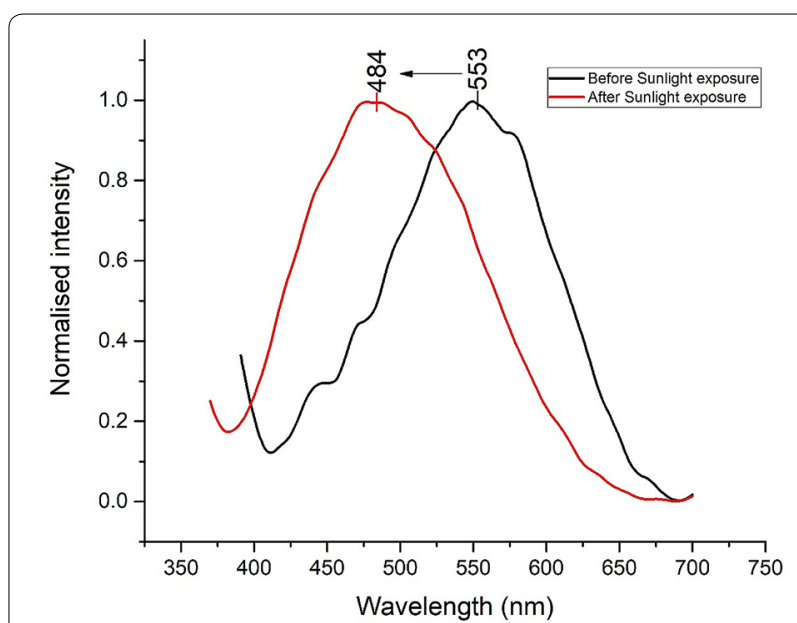

Fig. 9 Bleaching of yellowed linseed oil after $8 \mathrm{~h}$ exposure to sunlight

and stand oil hardly shows any yellowing. Poppy seed oil reaches a maximum fluorescence emission at $530 \mathrm{~nm}$ as a result of yellowing, while stand oil remains at $510 \mathrm{~nm}$ (Additional file 1: Figures S5, S6 for poppy seed oil and Additional file 1: Figures S7, S8 for stand oil). WLO takes longer to cure, but once cured, yellows much more rapidly than $\mathrm{LO}$, reaching its maximum fluorescence emission at $570 \mathrm{~nm}$ (the same as LO; Additional file 1: Figures S9, S10). During the curing process of all oils, no noticeable yellowing occurred, whereas once cured, the rate of change in yellowing and fluorescence were a result of the aging mechanism employed. 
The two-step process in fluorescence development was also observed in the colour changes, whereby the first incline lead to a yellow (Fig. $5 \mathrm{a}-\mathrm{d}$ ) and the second to a brown discolouration (Additional file 1: Figure S4). The brown discolouration became evidently visible with the discolouration from ammonia vapour, but occured over several months in all samples. A shorter wavelength fluorescence (blue, $460 \mathrm{~nm}$ ) indicates a yellow discolouration, while a longer wavelength (green or yellow; 500-550 nm) indicates that the oil has started to turn brown in colour. Although these observed changes were drastic, they are not unknown- paintings degrade over time [28, 62], which leads to colour changes. These colour changes can be a result of varnish discolouration [24], pigment degradation [16-18], or binder discolouration [26-28]. Pigments are commonly used for colour reconstructions, as they have been found to be irreversible [63]. Varnish is also known to be a major factor in the discolouration of paintings due to its yellowing tendencies and the formation of micro-cracks which turn the varnish a milky colour [25]. This can be accounted for by having a conservator remove the varnish layer. However, the process to determine the original colour of the binding medium is not as easily achieved, due to the cyclic nature of the discolouration.

Drying oils are found to bleach in light, whereas dark conditions accelerate discolouration. As there is no measure of the amount of 'darkness' a painting receives, the measure of the discolouration of binder is often misinterpreted. By relating the discolouration of drying oils to the fluorescence, the discolouration of drying oils can be accurately calculated regardless of the age of the painting or the light conditions to which it has been exposed.

\section{Conclusion}

The fluorescence of drying oils is largely dependent on the physical state of the oil. Liquid oils fluoresce in the ultra-violet range, and therefore cannot be seen by the naked eye. Cured oils fluoresce in the blue range, and shift to green (and sometimes yellow) fluorescence, as the oil yellows. Contrary to previous findings it is proposed that the fluorescence of drying oils is a result of subfluorophores which are poorly fluorescent in the liquid phase but become fluorescent once immobilised in the cured oil matrix. The bathochromic shift in fluorescence (from blue to green) is a result of increased rigidity in the cured oil matrix. The yellowing which is linked to the fluorescence of the oil is not a result of age, but rather of storage conditions, as was identified by the extreme storage conditions used in this study. Exposure to ammonia vapour rapidly increases the degree of yellowing, and therefore ammonia-based cleaning products should be avoided in museum environments, as the discolouration affects the aesthetic value of artworks. Light bleaches the oil and thus reverses the fluorescence shift, while dark storage conditions promote yellowing and fluorescence changes. Although light bleaches the yellowing of drying oils, this is not advised as it could simultaneously cause irreversible photodegradation of certain pigments.

The yellow discolouration of oils is not always visible when pigments are present, and in mixtures with non-fluorescent pigments, the fluorescence can be used to identify the degree of discolouration. The fluorescence peak colour provides a measure of yellowing and can therefore, be used to do digital colour corrections of paintings. As fluorescence spectroscopy is non-destructive, it can be used to determine the extent of yellowing without the need of sampling and is thus an advisable method for the monitoring of discolouration in paintings.

\section{Supplementary information}

Supplementary information accompanies this paper at https://doi. org/10.1186/s40494-020-00403-1.

Additional file 1. Supplementary information.

\section{Abbreviations}

LO: Linseed oil; WLO: Water-miscible linseed oil; SO: Stand oil; PO: Poppy seed oil; $\lambda_{\mathrm{em}}$ : Emission wavelength; $\lambda_{\mathrm{ex}}$ : Excitation wavelength.

\section{Acknowledgements}

The authors would like to thank Isabelle McGinn and Maggi Loubser from the Tangible Heritage Conservation program at the University of Pretoria for their continual support throughout this project. The authors would also like to acknowledge and thank Dr Mamoalosi Selepe for assisting with HPLC analyses and Daniel Pretorius' contribution in editing the manuscript.

\section{Authors' contributions}

The whole database construction and analysis presented in the manuscript had been achieved by the first author. The second author provided samples and equipment and made significant contributions to the content, reviewed the whole text and made valuable comments and suggestions. All authors read and approved the final manuscript.

\section{Funding}

The authors confirm that they are not currently in receipt of any research funding relating to the research presented in this manuscript.

\section{Availability of data and materials}

The dataset generated and analysed during the current study is available from the corresponding author on reasonable request.

\section{Competing interests}

The authors declare that they have no competing interests.

Received: 18 February 2020 Accepted: 11 June 2020

Published online: 19 June 2020

\section{References}

1. Izzo FC. 20th century artists' oil paints: a chemical-physical survey. (Thesis) 2011.

2. Poth U. Drying oils and related products. Ullmann's Encyclopedia of Industrial Chemistry. 2001. 
3. Van den Berg J. Analytical chemical studies on traditional linseed oil paints. (Thesis) 2002.

4. White R. The organic chemistry of museum objects. London: Buttersworth; 1994.

5. Van Dam EP, van den Berg KJ, Gaibor ANP, van Bommel M. Analysis of triglyceride degradation products in drying oils and oil paints using LCESI-MS. Int J Mass Spectrom. 2017;413:33-42.

6. Colombini MP, Modugno F. Organic mass spectrometry in art and archaeology. Hoboken: Wiley; 2009

7. Van der Doelen GA, van den Berg KJ, Boon JJ. Comparative chromatographic and mass-spectrometric studies of triterpenoid varnishes: fresh material and aged samples from paintings. Stud Conserv. 1998:43(4):249-64.

8. Levison HW. Yellowing and bleaching of paint films. Journal of the American Institute for Conservation. 1985;24(2):69-76.

9. Smith R. The artist's handbook. London: Dorling Kindersley; 1990.

10. Schilling MR, Mazurek J, Learner TJ. Studies of modern oil-based artists' paint media by gas chromatography/mass spectrometry. Modern paints uncovered: proceedings from the modern paints uncovered symposium, Getty Conservation Institute symposium proceedings series Getty Conservation Institute, Los Angeles; 2007: 129-139.

11. Foster A. The performance and properties of artisan water mixable oil colour compared with other oil-based paints by Winsor \& Newton. Modern paints uncovered: proceedings from the modern paints uncovered symposium; 2007: 53-57.

12. Carlyle L. Historical reconstructions of artists' oil paint: an investigation of oil processing methods and the use of selected artists' mediums. Deterioration of artists' paints: effects and analysis A joint meeting of ICOM-CC working groups $1 \& 2$ and the Paintings Section, UKIC, British Museum, London, September 10-11, 2001 Extended abstracts of publications; 2001.

13. Carlyle L. Historically accurate reconstructions of oil painters' materials: an overview of the Hart project 00-005. Reporting Highlights of the De Mayerne Programme,(Jaap J Boon and Esters SB Ferrera), Den Haag. 2006:63-76.

14. Katkade M, Syed H, Andhale R, Sontakke M. Fatty acid profile and quality assessment of safflower (Carthamus tinctorius) oil. J Pharmacogn Phytochem. 2018;7:3581-5.

15. Coşge B, Gürbüz B, Kiralan M. Oil content and fatty acid composition of some Safflower (Carthamus tinctorius L.) varieties sown in Spring and Winter. Int J Nat Eng Sci. 2007;1(3):11-5.

16. Kirchner E, van der Lans I, Ligterink F, Geldof M, Ness Proano Gaibor A, Hendriks E, et al. Digitally reconstructing van Gogh's field with irises near Arles. Part 2: pigment concentration maps. Color Res Appl. 2018;43(2):158-76.

17. Kirchner E, van der Lans I, Ligterink F, Geldof M, Megens L, Meedendorp T, et al. Digitally reconstructing Van Gogh's Field with Irises near Arles part 3: determining the original colors. Color Res Appl. 2018;43(3):311-27.

18. Monico L, Janssens K, Miliani C, Brunetti BG, Vagnini M, Vanmeert F, et al. The degradation process of lead chromate in paintings by Vincent van Gogh studied by means of Spectromicroscopic methods. 3: synthesis, characterization and detection of different crystal forms of the chrome yellow pigment. Anal Chem. 2012:85:851-9.

19. Centeno SA, Mahon D. The chemistry of aging in oil paintings: metal soaps and visual changes. Metrop Mus Art Bull. 2009;67(1):12-9.

20. Centeno SA, Hale C, Carò F, Cesaratto A, Shibayama N, Delaney J, et al. Van Gogh's Irises and Roses: the contribution of chemical analyses and imaging to the assessment of color changes in the red lake pigments. Herit Sci. 2017;5(1):18.

21. Shimadzu Y, Van Den Berg K. On metal soap related colour and transparency changes in a 19th C painting by Millais. Reporting Highlights of the De Mayerne Programme. Netherlands Organisation for Scientific Research (NWO); 2006. P. 43-52.

22. Hermans J, Osmond G, van Loon A, ledema P, Chapman R, Drennan J, et al. Electron microscopy imaging of zinc soaps nucleation in oil paint. Microsc Microanal. 2018:24(3):318-22.

23. Hermans JJ, Keune K, van Loon A, Corkery RW, ledema PD. Ionomer-like structure in mature oil paint binding media. RSC Adv. 2016;6(96):93363-9.

24. Kirchner E, der Lans I, Ligterink F, Hendriks E, Delaney J. Digitally reconstructing van Gogh's field with irises near Arles. Part 1: varnish. Color Res Appl. 2018;43(2):150-7.
25. Nicolaus K. Restoration of Paintings. New York: Konemann; 1999.

26. Mallégol J, Lemaire J, Gardette J-L. Yellowing of oil-based paints. Stud Conserv. 2001:46:121-31.

27. Privett O, Blank M, Covell J, Lundberg W. Yellowing of oil films. J Am Oil Chem Soc. 1961;38(1):22-7.

28. Geldof M, Gaibor ANP, Ligterink F, Hendriks E, Kirchner E. Reconstructing Van Gogh's palette to determine the optical characteristics of his paints. Herit Sci. 2018;6(1):17.

29. de la Rie ER. Fluorescence of paint and varnish layers (Part II). Stud Conserv. 1982;27(2):65.

30. Chio K, Tappel AL. Synthesis and characterization of the fluorescent products derived from malonaldehyde and amino acids. Biochemistry. 1969;8(7):2821-7.

31. Franks F, Roberts B. Quantitative study of the oxidative discoloration of ethyl linoleate. I. Oxidation in the bulk phase. J Appl Chem. 1963;13(7):302-9.

32. O'Neill L, Rybicka S, Robey T. Yellowing of drying oil films. Chemistry and industry. 1962; 1796-7.

33. Rakoff H, Thomas F, Gast L. Reversibility of yellowing phenomenon in linseed-based paints. J Coat Technol. 1979;51(649):25-8.

34. Mounier A, Belin C, Daniel F. Spectrofluorimetric study of the ageing of mixtions used in the gildings of mediaeval wall paintings. Environ Sci Pollut Res. 2011;18(5):772-82

35. loakimoglou E, Boyatzis S, Argitis P, Fostiridou A, Papapanagiotou K, Yannovits $N$. Thin-film study on the oxidation of linseed oil in the presence of selected copper pigments. Chem Mater. 1999;11(8):2013-22.

36. Kumarathasan R, Rajkumar AB, Hunter NR, Gesser HD. Autoxidation and yellowing of methyl linolenate. Prog Lipid Res. 1992;31(2):109-26.

37. Bayliss S, van den Berg KJ, Burnstock A, de Groot S, van Keulen H, Sawicka A. An investigation into the separation and migration of oil in paintings by Erik Oldenhof. Microchem J. 2016;124:974-82.

38. Cosentino A. Practical notes on ultraviolet technical photography for art examination. Conservar Património. 2015. https://doi.org/10.14568/ cp2015006.

39. Pelagotti A, Pezzati L, Bevilacqua N, Vascotto V, Reillon V, Daffara C, editors. A study of UV fluorescence emission of painting materials. Art '05-8th International Conference on Non-Destructive Investigations and Microanalysis for the Diagnostics and Conservation of the Cultural and Environmental Heritage Lecce, Italy; 2005.

40. Rorimer JJ. Ultra-violet rays and their use in the examination of works of art. New York: Metropolitan Museum of Art; 1931.

41. Mounier A, Lazare S, Le Bourdon G, Aupetit C, Servant L, Daniel F. LEDuSF: a new portable device for fragile artworks analyses. Application on medieval pigments. Microchem J. 2016;126:480-7.

42. Romani A, Clementi C, Miliani C, Favaro G. Fluorescence spectroscopy: a powerful technique for the noninvasive characterization of artwork. Acc Chem Res. 2010;43(6):837-46.

43. Romani A, Clementi C, Miliani C, Brunetti B, Sgamellotti A, Favaro G. Portable equipment for luminescence lifetime measurements on surfaces. Appl Spectrosc. 2008;62(12):1395-9.

44. Fuster-López L, Stols-Witlox M, Picollo M. UV-Vis Luminescence imaging techniques/Técnicas de imagen de luminiscencia UV-Vis. Colección Conservation $360^{\circ} .2020$

45. Comelli D, Valentini G, Nevin A, Farina A, Toniolo L, Cubeddu R. A portable UV-fluorescence multispectral imaging system for the analysis of painted surfaces. Rev Sci Instrum. 2008;79(8):086112.

46. Pavia DL, Lampman GM, KRIS G. Introduction to spectroscopy Third Edition. Thomson Learn Inc. 2001;15:579.

47. Van den Berg JD, van den Berg KJ, Boon JJ. Identification of non-crosslinked compounds in methanolic extracts of cured and aged linseed oil-based paint films using gas chromatography-mass spectrometry. J Chromatogr A. 2002;950(1-2):195-211.

48. Udell NA, Hodgkins RE, Berrie BH, Meldrum T. Physical and chemical properties of traditional and water-mixable oil paints assessed using single-sided NMR. Microchem J. 2017;133:31-6.

49. Lakowicz JR. Principles of fluorescence spectroscopy. New York: Plenum Press; 1983.

50. Sutherland K. Solvent-extractable components of linseed oil paint films. Stud Conserv. 2003;48(2):111-35. 
51. Zhu S, Song Y, Shao J, Zhao X, Yang B. Non-conjugated polymer dots with crosslink-enhanced emission in the absence of fluorophore units. Angew Chem Int Ed. 2015;54(49):14626-37.

52. Bonaduce I, Duce C, Lluveras-Tenorio A, Lee J, Ormsby B, Burnstock A, et al. Conservation issues of modern oil paintings: a molecular model on paint curing. Acc Chem Res. 2019;52(12):3397-406.

53. Bonaduce I, Carlyle L, Colombini MP, Duce C, Ferrari C, Ribechini E, et al. New insights into the ageing of linseed oil paint binder: a qualitative and quantitative analytical study. PLoS ONE. 2012;7(11):e49333.

54. Schaich K. Lipid Oxidation: New Perspectives on an Old Reaction. Bailey's industrial oil and fat products. 2020.

55. Schaeffer TT. Effects of light on materials in collections: data on photoflash and related sources. Los Angels: Getty Publications; 2001.

56. Feller RL. Accelerated aging: photochemical and thermal aspects. Los Angels: Getty Publications; 1995.

57. Comelli D, Nevin AB, Verri G, Valentini G, Cubeddu R. Time-resolved fluorescence spectroscopy and fluorescence lifetime imaging for the analysis of organic materials in wall painting replicas. 2015

58. Larson LJ, Shin K-SK, Zink Jl. Photoluminescence spectroscopy of natural resins and organic binding media of paintings. J Am Inst Conserv. 1991;30(1):89-104.
59. Miyoshi T. Fluorescence from oil colours, linseed oil and poppy oil under N2 laser excitation. Jpn J Appl Phys. 1985;24(3):371-2.

60. Itakura K, Uchida K. Evidence that malondialdehyde-derived aminoenimine is not a fluorescent age pigment. Chem Res Toxicol. 2001;14(5):473-5.

61. Carlyle L, Binnie N, Kaminska E, Ruggles A, editors. The yellowing/bleaching of oil paintings and oil paint samples, including the effect of oil processing, driers and mediums on the colour of lead white paint. Triennial meeting (13th), Rio de Janeiro, 22-27 September 2002: preprints; 2002.

62. Berns RS, Byrns S, Casadio F, Fiedler I, Gallagher C, Imai FH, et al. Rejuvenating the color palette of Georges Seurat's A Sunday on La Grande Jatte-1884: a simulation. Color Res Appl. 2006;31(4):278-93.

63. Miliani C, Monico L, Melo MJ, Fantacci S, Angelin EM, Romani A, et al. Photochemistry of Artists' Dyes and Pigments: towards better understanding and prevention of colour change in works of art. Angew Chem Int Ed. 2018;57(25):7324-34

\section{Publisher's Note}

Springer Nature remains neutral with regard to jurisdictional claims in published maps and institutional affiliations.

\section{Submit your manuscript to a SpringerOpen ${ }^{\circ}$ journal and benefit from:}

- Convenient online submission

- Rigorous peer review

- Open access: articles freely available online

- High visibility within the field

- Retaining the copyright to your article

Submit your next manuscript at $\boldsymbol{\nabla}$ springeropen.com 